\title{
Iov Hybrid Architecture - Inter-Vehicular Communication and Data Transmission
}

\author{
Ishan Yash, Samir Ahmed Talkal, Karapurkar Shivani Prashant
}

\begin{abstract}
The Internet of Vehicle has an Intelligent Vehicular Grid consisting of the sensor platform, which continuously processes the data gathered from the surrounding of the vehicle and feeds it to the infrastructure. This data is further utilized by other vehicles to assist in traffic management, vehicle localization, pollution control and safe navigation. In this paper, we have suggested the ways through which we could attain a high data rate, seamless connectivity, security, improved quality of service and scalability. The first section of the paper consists of the proposed architecture and its benefits, and the latter consists of the scope of improvement with results using sensor dataset its application and challenges. Communication between the infrastructure and other vehicles can create privacy and security violations; hence, we also address ways to ensure the location privacy of the user.

Keywords: IoV (Internet of Vehicles), VANET (Vehicular ad-hoc network), V2X, IoT (Internet of Things), SVM (support vector machine), RAU (Radio Access unit)
\end{abstract}

\section{INTRODUCTION}

The IoV is a joining of three systems: a between vehicle arrange, an intra-vehicle organize, and vehicular portable Internet. According to this concept, the IoV is a distributed system at an enormous scale dedicated to wireless communication and information exchange. The information exchange can be between the vehicle and $X$ where $X$ can be another vehicle, street, human and web, which is as per concurred correspondence conventions and information connection principles. This implementation can be a boon to integrated networks like intelligent traffic management, vehicle control and dynamic information services, representing a typical application of Internet of Things (IoT) technology in smart transportation system (ITS). ITS is relied upon to be broadly conveyed for the IoV worldview to help a wide assortment of use running from low information rate traffic control administrations to high information rate and deferral basic interactive media administrations. The ITS utilizes the coordination of sensors, locally available unit (OBU), and believed stage module to impart imperative data of the vehicle to the roadside unit [8], The assembly of innovation benefits data correspondences, natural assurance, vitality preservation, and security. To get the upper hand over others in this emerging market, the procurement of core technologies and standards will be critical to secure a strategic

Revised Manuscript Received on December 16, 2019.

Ishan Yash, Electronics and communications Engineering with specialization in IoT and sensors, VIT Vellore, Tamil Nadu, India, ishan.yash17@gmail.com

Samir Ahmed Talkal, Information Technology Engineering, VIT Vellore Tamil Nadu, India, samirtalkal@gmail.com

Karapurkar Shivani Prashant, Electrical and Electronics Engineering, VIT Vellore Tamil Nadu, India, shivonpk@gmail.com advantage. However, the combination of the IoV with various frameworks need to have a similar need as that of working of the IoV advances themselves. As a result of this, the IoV may come to be a fundamental piece of the most noteworthy Internet of things (IoT) foundation by method for its consummation. Here, it must be underlined as essential, that the subsequent stage in IoV advancement will be the joint effort and interconnection between the transportation division and different areas. [1].

\section{EVOLUTION OF IOV}

The advanced armada of vehicles is developing from an assortment of sensor stages that give data to the drivers and transfer the adjusted sensor information which has significance, on the web which has the system of self-sufficient vehicles, that trade their sensor contributions among one another to enhance a few distinctive utility capacities. The development in the vehicle armada is fairly like what we saw 10 years prior in the sensor area, to sensor web and Internet of Things In the smart home, the IoT formed by the horde of sensors and actuators, that cowl the living arrangement inside and remotely, can deal with the entirety of the utilities in the most sensible way, with greatest solace to inhabitants, with only no human intercession. [2]

So also, in a cutting edge vitality lattice, IoT comprising of a wide range of segments can control stacks steadily and productively, with the administrators currently backing the capacity of witnesses. In the vehicular system, the Internet of Vehicles (IoV) is more many-sided than the smart home and smart vitality network IoTs.

\section{A. Difference Between IoT and IoV}

The properties what make IoVs significantly different from IoT are the management of mobility and wireless bottleneck, which also should guarantee motion privacy. IoV has safety-critical applications which have low latency requirements. The Security of the vehicle and safety from DDoS attacks are made possible by $\mathrm{V} 2 \mathrm{~V}$.

\section{RELATED WORK}

There is a gigantic number of various remote access advancements, proposed by the business and the exploration network, with regards to vehicular interchanges. They can comprehensively be seen into intra-vehicular, bury vehicular, and vehicle to framework correspondence. A huge assortment of innovations is accessible in the writing for every one of the classes referenced previously. Be that as it may, our place of center is the vehicle to foundation correspondence. A few access advances have just

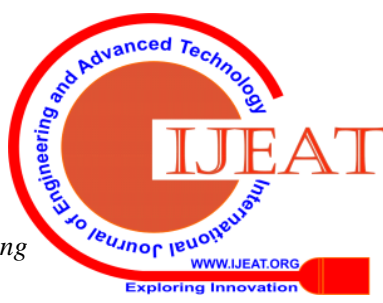


been presented and evaluated in the setting of VANET, (for example, WLAN) [23], Worldwide Interoperability for Microwave Access (WiMAX)[24], and cell technologies such as4G/LTE[21]).

Differential security has been proposed as a segment to furtively share data to such a degree, that anything that can be instructed if a particular data information proprietor is joined into the database can in like manner be found out if the specific data owner is avoided in the database. To achieve this protection, guarantee differential security arranges that only a sublinear number of requests approach the database and that uproar comparing to the overall affectability of checking examination is incorporated (liberated from the quantity of information proprietors).

Distributional security is an insurance framework which says that the complete discharged information reveals the concealed ground truth course. This verifies singular data proprietors and is cautiously more grounded than differential protection. Be that as it may, it is computationally wasteful however can work over a huge class of inquiries known as Vapnik-Chervonenkis (VC) measurement, which is one estimation of learning theory as for AI.

Zero-information protection is a cryptographically influenced security definition that is cautiously more grounded than differential protection. Crow mixing protection is flimsier than differential security; nevertheless, with a pre-analyzing step, it satisfies both differential protection and zero-information protection. These systems do exclude commotion direct in the quantity of information proprietors and rely upon forceful looking at, which unfavorably impacts the precision estimations.[13]

\section{PROPOSED MODEL}

This section consists of the system model for the proposed solution, which uses multiple access technologies to enable uninterrupted communication in IoV, which also targets V2I communication. The access technologies chosen are WAVE [9], long-range $\mathrm{WiFi}$ and 4G/LTE.

The typical IoV architecture consists of three layers as shown in Fig 1.0 [10]

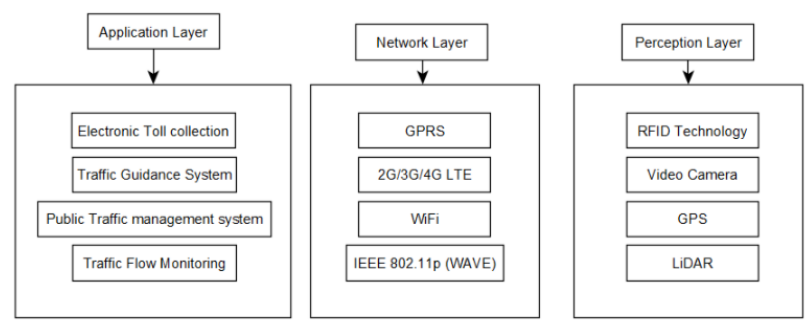

Fig 1. - Architecture of IoV

The proposed design pursues a three-layered methodology so as to disentangle the usefulness of different parts. The base most layer, which is the customer level spreads bury and intravehicular correspondence, this is the place the vehicles are outfitted with the vehicular GID terminal and different sensors, for example, GPS and LiDAR. The Connection level arrangements with the interconnectivity of different system segments inside the system and reconciliation of other accessible frameworks inside a vehicular domain. At long last, the cloud level is liable for empowering all the IoV administrations like virtualization, real-time interaction, security, data transfer and storage. Fig 2.0 is a generalized view of proposed hybrid IoVs architecture.

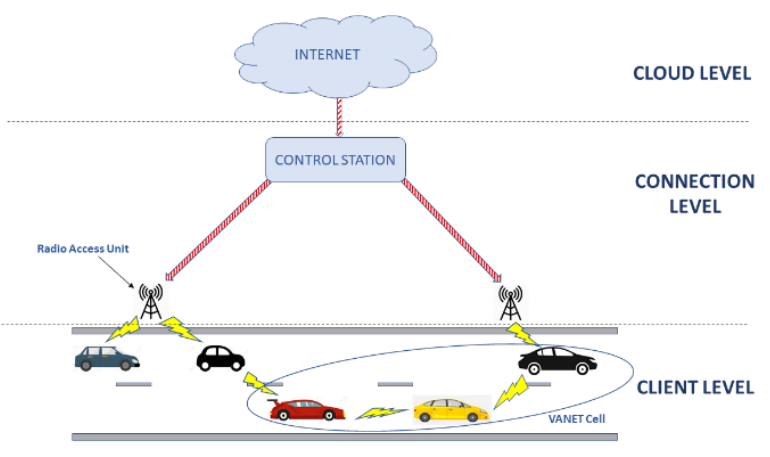

Fig 2.0 - Hybrid VANETs architecture.

The Control framework or system is another essential part that is liable for controlling the remainder of the tasks of this engineering. The primary elements of the framework, for example, recurrence allotment, adjustment/demodulation, and handling, are performed at the focal site, which in the long run improves the structure of the Radio Access Unit. The unified design permits a unique configuration of radio asset and limit allotment. The optical fiber is straightforward to tweak, radio recurrence, and bit rate; thus, various administrations on a solitary multimode fiber can be bolstered simultaneously utilizing RoF oversaw by the framework. [8]

Notwithstanding the previously mentioned, we can likewise utilize DSRC reference points to assist safe with removing keeping inside the unit. Automakers, be that as it may, have been against utilizing V2V or V2I to execute security plans for customers. The essential explanation is a weakness if there should arise an occurrence of mishaps [13]. State, expect the vehicle in front perniciously advises my vehicle to drag on account of an abrupt obstruction, while there is no boundary. My vehicle ends and is slammed with the vehicle behind. I am actually at fault.

This issue is settled utilizing Standard Adaptive Cruise Control (ACC) and Cooperative Adaptive Cruise Control (CACC) company control frameworks. The Cooperative Adaptive Cruise Control is a continuation of the versatile journey control idea. CACC perceives longitudinal computerized vehicle control. Supplementing the input circle utilized in the ACC, which works with Radar or LIDAR calculations to get acquainted with the range to the vehicle in front, the speeding up of the first vehicle is utilized in a feed-forward circle. The speeding up of that vehicle is acquired from the Cooperative Awareness Messages (BSM in the US) it sends the information utilizing ETSI ITS-G5 or DSRC/WAVE innovation (in light of IEEE 802.11p). Commonly, these messages are transmitted a few occurrences with regards to second by future vehicles furnished with ITS capacities. [30][31] 


\section{A. Comparison Between The Predefined And The Proposed Model Of Architecture}

The affecting factors which are made into consideration are Throughput with respect to time, Delay, Impact of mobility speed on throughput and Impact of server load as for versatility speed. The proposed engineering with dynamic and versatile system determination can beat WAVE and long-extend WiFi norms. In spite of the fact that proposed half breed design chooses the best system interface relying upon the accessibility around then regarding a few parameters however, overwhelming client applications, for example, Voice over Internet Protocol and video conferencing, needs ceaseless transmission and are consistently engaged, and in this way the throughput conduces to go down beneath to bring down qualities in the end, in every one of the cases. All things considered, the proposed engineering is anticipated to stay in the main job when contrasted with different partners, as referenced in [8]. The principle factor behind darkened throughput is the ascent in versatility speed of the source and goal vehicles during correspondence. The half and half engineering will have the option to adapt well to developing versatility speed as related to different alternatives because of changing interface choice dependent on use prerequisite. [8]

\section{B. Security Problem: Privacy Violations In V2I Communications}

The Centralized route framework has security issues and can prompt numerous protection infringement. The gathered information can be used by the route servers to follow clients and find their ways and other private information. Normally, Service Providers (like Google and UBER) are focused on securing client protection. Be that as it may, security ensures were much of the time broken inside the past (deliberately or accidentally). Instances of security infringement with WAZE. In the WAZE Privacy Attack, WAZE enables removed clients to see current traffic in a subjective window. By moving the window, the interloper tracks the objective. In the WAZE DDoS Attack, the vindictive client mirrors a case which shows various WAZE vehicles in a little territory, giving a bit of bogus data by reenacting traffic bottleneck. While we focus on the Privacy infringement issue, an issue exceptionally regular to all applications that use versatile information from IoT or IoV to Servers in the Cloud. We will present a proficient, adaptable answer for the above issue, which is Haystack. [13]

\section{Implementation Of Haystack Privacy}

In Haystack, First, the information proprietors have the high ground of locally privatizing their own information, liberated from other information proprietors or any incorporated administrations, which may end up being a path for any security break. Second, information proprietors blend in with, and are indistinguishable, from in any event 'c' swarms, where each group is framed of at any rate ' $\mathrm{k}$ ' information proprietors. At long last, information proprietors play out a cryptographic mystery compose with the end goal that no aggregator knows where in the information structure an information proprietor referenced to. Viably, in laymen words, every datum proprietor can be picturised as "sneaking in a bundle".
The essential commitment of Haystack Privacy is that everybody in the group locks in. For instance, on the off chance that we publicly support vehicle densities crosswise over Vellore City utilizing the question, for example, "Would you say you are at VIT?". An information proprietor (vehicle) begins by reacting to the question "Am I at VIT?". Earlier work utilizing the Laplace component [29], [28] would have everybody at VIT answer truthfully. Then, a minimal amount of privacy noise is added to shield privacy.

In Haystack Privacy, every datum proprietor reacts to the inquiry referenced previously. A little division of those not at VIT will answer "Indeed, I'm at VIT". A little portion at VIT will react "No, I'm not at VIT". The two cases give sensible deniability and are controlled by two diverse Bernoulli preliminaries determined by the question. To evaluate the inexact tally, the normal estimation of the protection clamour because of the Bernoulli preliminaries is expelled in the wake of computing. One thought is that the quantity of individuals at VIT is fixed. While the quantity of clients in any territory (e.g., Katpadi) might be set, the consideration of contributions from the clients not at that area empowers us to use the law of enormous numbers to guarantee the area protection of the client and that the assessed security clamour moves toward the normal worth and in this manner improves exactness. The nitty gritty of sheaf security's system is referenced in [13].

\section{BENEFITS OF IOV MODEL}

The examination and headway, just as the mechanical use of IoV advancements, will profit the mix of car and data innovation. The consolidated data administrations of vehicles, vehicle wellbeing, and financial execution will add to a progressively shrewd urban transportation framework and a superior social and monetary improvement. The IoV will have sweeping significance on the shopper vehicle showcase, buyer way of life, and even methods of conduct. The up and coming IoV market will see quickened development in the Asian-Pacific locale. McKinsey Global Institute declared in June 2013 that the "IoT can possibly drive around \$6.2 trillion in new worldwide financial worth yearly by $2025 "$. Eighty to Hundred percent of all makers will apply IoT innovation by at that point, prompting a potential financial effect of $\$ 2.3$ trillion for the worldwide assembling industry. As indicated by the information on the APEC site, the part nations share roughly 55 percent of the world's GDP. In laymen words, APEC individuals will be developing by $\$ 3.41$ trillion in GDP and producers of the economies will include $\$ 1.27$ trillion development in the in the interim. This can be a help to the makers and the up and coming analysts. [1]

\section{A. Iov Model As A Cost-Effective Model}

The expense effectiveness is of the most noteworthy noteworthiness in the multi-interfaced engineering. The eff ort was to make the structure sensible by picking less expensive RAUs following an extremely simple transmission component. It is pertinent to make reference to that the expenses might be higher in the zones where the fiber should be set without any preparation. The proposed RoF approach is sturdier than the current designs

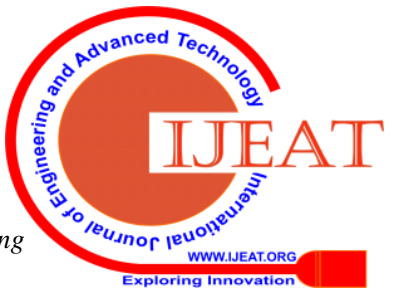


as far as information rate, transfer speed accessibility, and nature of administration arrangement. The general cost factor relies upon the current foundation accessible. For instance, if the proposed architecture is to be deployed in an area already covered by fibre services, the only significant cost can be the RAU deployment, which can be costly.

\section{B. Congestion Control}

Congestion on the system is one of only a handful barely any disturbing perspectives that may continuously prompt lull the execution of the general system. Mishaps, crises, or different accidents typically cause this clog in VANET as a solitary purpose of disappointment; the whole system gives off an impression of being a bottleneck and goes down. As the proposed engineering underpins numerous interfaces, so if there is some issue with one interface, different hubs can carry on their correspondence by some different interfaces.

\section{Support For Future Technologies}

The proposed framework shows its similarity to help numerous future advancements, (for example, Fifth Generation (5G) or HaLow) [32] as the RAU configuration can bolster a wide scope of recurrence groups independent of the remote innovation standard. In this way, the conveyed RAUs along the roadways can serve to tune in on different recurrence channels without dreading innovation oldness.

\section{Ease Of Management}

The regions where fiber is starting at now sent, the proposed plan can be completed rapidly with least control system. Scarcely any CS are adequate to give the structure the board office on account of the plausibility of fiber accessibility at the backhaul, and CS is the primary united component for all kind of dealing with on the customer requests.

\section{E. Carbon Footprint Savings}

According to the measurements, ICTis represented $2 \%$ of the worldwide carbon impressions, and this pattern is going to proceed with a yearly increment of $10 \%$ [30]. Each exertion made to limit this impact would in the long run avert the earth. The proposed engineering utilizes fiber at the backhaul to associate with the system spine. Henceforth, it would add to the carbon emanation investment funds towards the marvel of Green Networks [33].

\section{MAINTENANCE AND IMPROVEMENTS}

Due to the enormous overhead caused for course revelation and course upkeep for exceptionally versatile clumsy vehicles, not many of the current directing conventions for between vehicle systems can deal with the weights on wellbeing applications $[14,15]$. A significant piece of steering conventions for specially appointed systems is basically founded on topology and wishes the built up request of a start to finish course between the stock and the goal before sending any data bundle. Because of quick alterations inside the system topology and profoundly different correspondence trade channel conditions, the start to finish ways which are controlled by customary impromptu topology-based directing conventions are effectively broken. To take care of this issue, a few steering conventions have been proposed [16,17] [18,19] [20] [21]. For instance, Namboodiri and Gao [16] proposed an expectation based steering for VANETs. The $\mathrm{PBR}$ is an unconstrained directing convention, which is specifically custom fitted to the parkway versatility situation, to enhance steering capacities without utilizing the overhead of a proactive convention. The PBR abuses the deterministic movement example and rates, to foresee generally to what extent a current course between a "hub" vehicle and a "passage" vehicle will last. Utilizing this forecast, the creators pre-emptively make new courses before the current course lifetime lapses. Toutouh et al. [14] proposed an outstanding portable impromptu system directing convention for VANETs to enhance parameter settings for interface state steering by utilizing a programmed advancement instrument. Nzounta et al. [19] proposed a class of street based VANET steering conventions. These conventions influence constant vehicular traffic data to make ways.

Besides, topographical sending permits the use of any hub on a street fragment to move bundles between two back to back convergences on the course, lessening the way's responsiveness to singular hub advances. Huang et al. [20] inspected the proficiency of hub disjoint way directing subject to various degrees of way coupling, with and without parcel excess. An Adaptive Approach for Information Dissemination (AID) in VANETs was displayed in [18], in which every hub accumulated the data on neighbor hubs, for example, separation estimations, fixed upper/lower limits and the quantity of neighboring hubs. Utilizing this data, every hub progressively changes the estimations of neighborhood parameters. The creators of this methodology likewise proposed a rebroadcasting calculation to get the edge esteem. The outcomes acquired show that AID is superior to other customary plans in its classification. Fathy et al. [21] proposed a QoS Aware convention for improving QoS in VANET. The convention utilizes Multi-Protocol Label Switching (MPLS), which runs over any Layer 2 innovations; and switches forward bundles by taking a gander at the mark of the parcel without looking the steering table for the following bounce.

\section{A. Improving The Data Processing, Transfer And Outcome Prediction By Using A Simple Machine Learning Model.}

An approach for better prediction of time series data acquired from the sensors attached in the vehicle. This will aid the memory usage and computation time for the predictability of the outcomes or the labels in the model that we train using machine learning. We will be using LIBSVM [25] with the default settings, for the training of the model. We used Wall-Following Robot Navigation Sensor Data, Data Set [26].

Our motive was to generalize the data processing and prediction of the data which we have acquired through the sensors attached. The information is in the form of time series which will be used as the feature and the outcomes deciding the movement will be used as the label. This can be implemented on any data set,

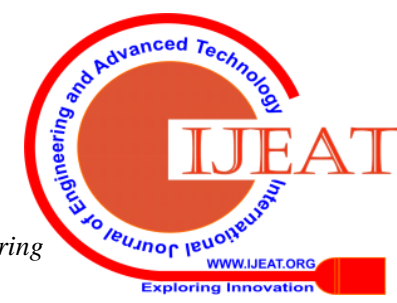


which is in the form of time-series, and the outcomes can further be predicted.

Experimentation:

We used three different methods for training and predicting the labels of the input features, out of which the LIBSVM gave the best accuracy in minimal time. Fig 3 shows the comparison between the methods used.

In Fig3, Series 1 is the accuracy of the particular method, and Series2 is the test size of the dataset. The code to implement the below-mentioned results is available at [27].

B. Dynamic Time Warping Distances As Features For Improved Time Series Classification

An easy method for classification of time series that exploits DTW's fervor on this job. However, rather than directly using DTW to find nearest neighbors as a distance measure, the method uses DTW to make new highlights which are then sent to a standard machine learning technique. It is shown after experiments that the method makes better over one-nearest neighbor DTW on 31 out of 47 UCR time series benchmark datasets [35]. Also, this technique can be easily continued to be in use in sequence with other techniques.[34]

\section{Support-Vector Machine}

In machine learning, support vector machines (SVMs) are supervised learning models with established algorithms for learning that examine data which is used for regression analysis and classification. [36]

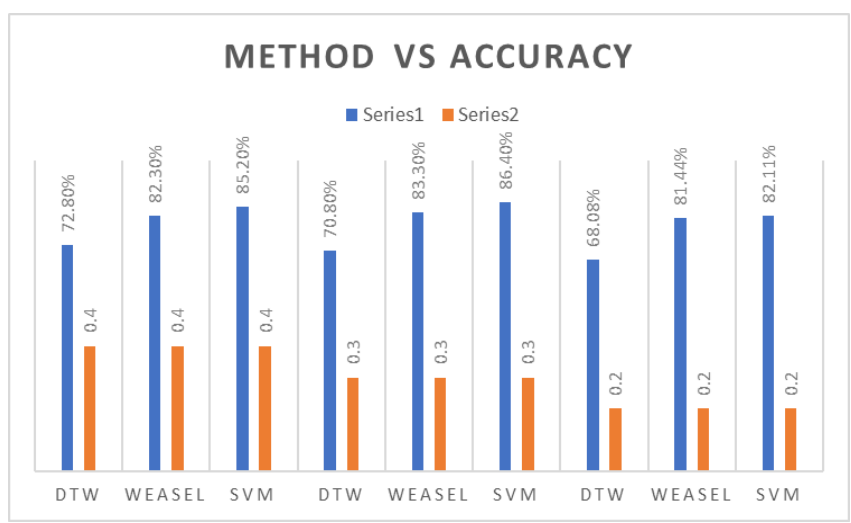

Fig 3

Below is the tabular representation of the accuracies on the various test sizes.

\begin{tabular}{|c|c|c|c|}
\hline \multirow{2}{*}{$\begin{array}{l}\text { Method } \\
\text { Used }\end{array}$} & \multicolumn{3}{|c|}{ Table Column Head } \\
\hline & $\operatorname{Accuracy}(\%)$ & Test size & Dataset \\
\hline DTW & $\begin{array}{ll}72.8 & , 70.8, \\
68.08 & \end{array}$ & $\begin{array}{l}0.4,0.3,0 . \\
2\end{array}$ & $\begin{array}{l}\text { sensor_readi } \\
\text { ngs_24 }\end{array}$ \\
\hline WEASEL & $82.3,83.3,81.4$ & $\begin{array}{l}0.4,0.3,0 . \\
2\end{array}$ & $\begin{array}{l}\text { sensor_readi } \\
\text { ngs_24 }\end{array}$ \\
\hline SVM & $85.2,86.4,82.1$ & $\begin{array}{l}0.4,0.3,0 . \\
2\end{array}$ & $\begin{array}{l}\text { sensor_readi } \\
\text { ngs_24 }\end{array}$ \\
\hline
\end{tabular}

Table 1

The Dataset has to be made suitable for the operation converting the commands to the numbers and then stored as labels. LIBSVM is used with default settings which will allow us not to be dependent on the alterations for the accuracy, too much.
Below are the plots showing the predicted classes and test labels as a plot for first one hundred, two hundred, three hundred and four hundred classes.

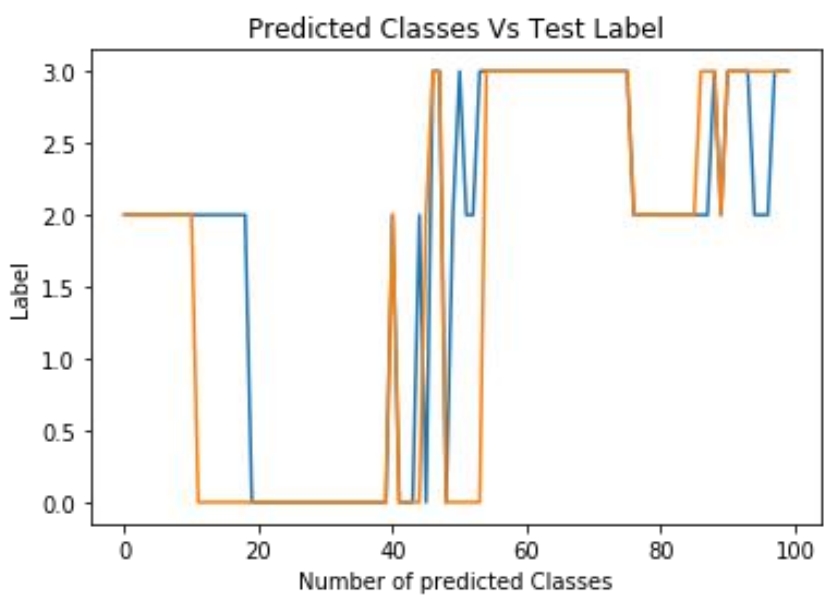

Fig 4 -First 100 labels

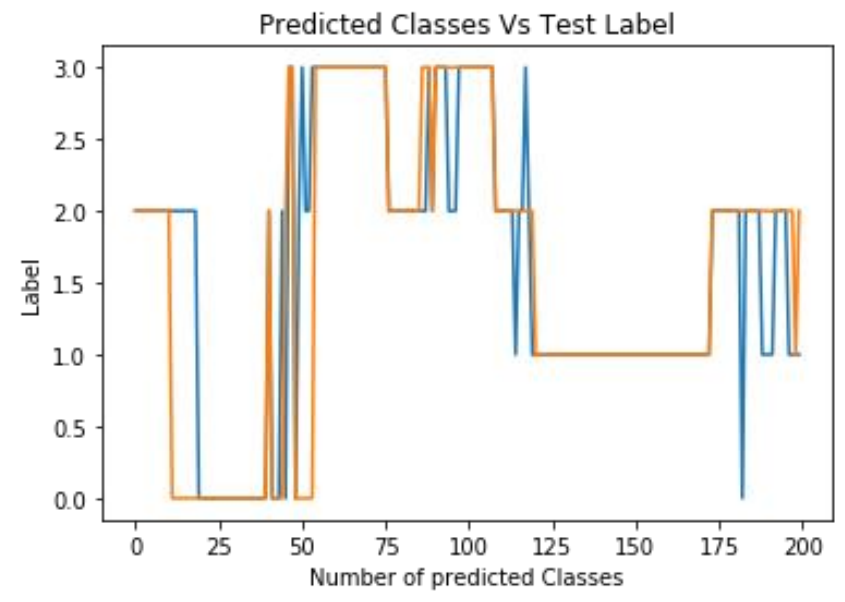

Fig 5 -First 200 labels

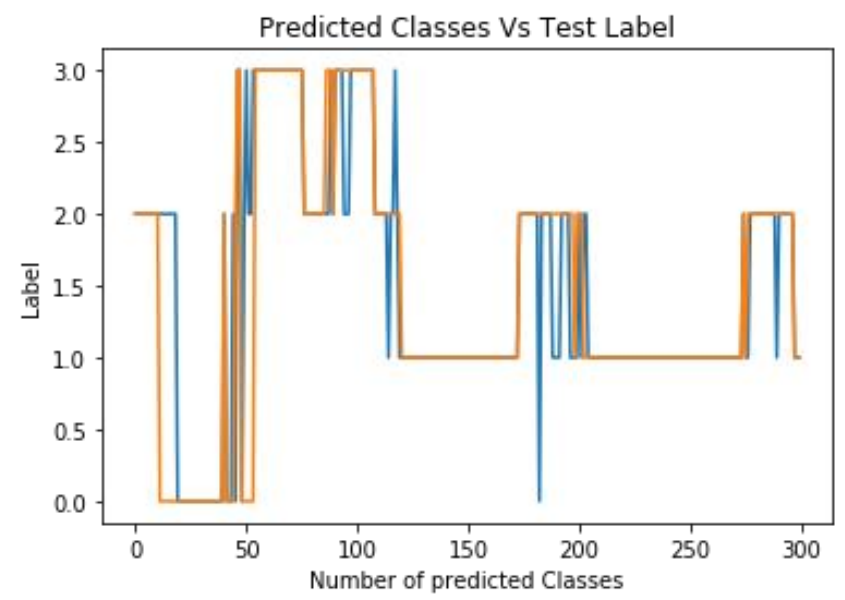

Fig 6 -First 300 labels 


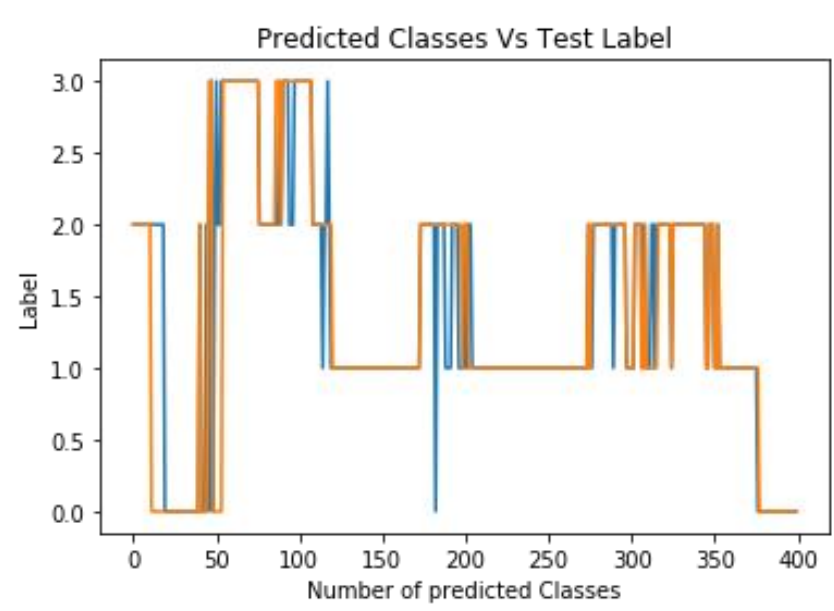

Fig 7 -First 400 labels

This shows us the improvement of the model in predicting the classes after successive intervals of each hundred classes.

This can be generalized, for any sensor data which is in the form of time series and can be implemented to train a model, which can efficiently predict any kind of outcomes for the data which needs to be transferred from one vehicle to another vehicle $(\mathrm{V} 2 \mathrm{~V})$ or from any device connected through the internet to the vehicle (V2X).

Code to implement the above results are available at [27]

\section{CHALLENGES}

Apart from having enormous opportunities, IoV has several obstacles to overcome, as well. Lack of coordination and communication between two users is the biggest challenge. A unique problem is the processing and storage of colossal information made in IoV because of the significant number of associated vehicles. For instance, driverless cars are relied upon to process about $1 \mathrm{~GB}$ of data for every second. Since IoV includes incorporating a wide range of technologies, services and standards, there is the requirement for information security. As an open system, public network, IoV is an objective for interruptions and cyber-attacks that may prompt physical harm and privacy leakages. The network hardware can malfunction. The system needs to manage off incorrect information, just as defective interchanges, for example, disavowal of administration assaults. In a circumstance where vehicles move quickly, and system topology continues evolving consistently, which is a challenge for keeping the nodes associated and provide the assets for transmitting and receiving in real-time. To quicken adoption, institutionalization and interoperability are crucial. Absence of standard makes compelling V2V communication troublesome. Embracing open norms will empower easy sharing of data. For the advancement of better implementation and to open global levels the Government ought to partake as well as urge ventures to team up.[11]

\section{APPLICATIONS}

IoV applications are diverse. IoV applications can be isolated into two noteworthy classifications: Safety applications and User applications. The applications which leads to the expansion of vehicle wellbeing and improvises the security of the travelers on the streets by advising the vehicles about any hazardous circumstances in the neighboring are called safety applications. The applications that offer some incentive included administrations are called User applications. Safe driving alludes to helpful crash shirking frameworks with the utilization of sensors to distinguish impending impact and gives cautioning to the driver. The application includes periodic status messages and rises messages. A message of crisis is activated by the development of occasion, for example, car influx, mishap, terrible street condition. Traffic control is another application in which IoV will realize essential changes to urban clog management, urban traffic, transport and co-ordinations, and our aggregate way of life.[11]

Innovations to upgrade vehicular and traveler security are of extraordinary intrigue, and one of the significant applications is a crash response. Connected automotive can naturally send ongoing information about an accident alongside the vehicle area to crisis groups. This can spare lives by quickening crisis reaction. To be explicit, collision warning incorporates notices about a chain auto collision, alerts about street conditions, for example, tricky street, and moving toward crisis vehicle cautioning. From one viewpoint, collision warning could be utilized to caution vehicles of a mishap that happened further along the road, in this way introducing a heap up from happening. Then again, they could likewise be utilized to give drivers early warning and keep a mishap from occurring in any case. Note that driving close and through crossing points is a standout amongst the most unpredictable difficulties that drivers face since at least two traffic streams meet, and the likelihood of a crash is high. The savvy convergence, where such regular traffic control gadgets as signs for traffic and stop signals are evacuated, has been an interesting territory of research for late years. Vehicles arrange their development over the crossing point through a blend of brought together and circulated continuous essential leadership, using worldwide situating, remote interchanges and in-vehicle detecting and computation. Various answers for crash avoidance of different vehicles at a crossing point have been proposed. A computationally effective control law has been gotten from misuse of the monotonicity of the vehicles' elements, yet it has not been connected to multiple cars.

The capacity to remotely get to a vehicle makes potential administrations, for example, remote entryway open and stolen vehicle recuperation. Associated vehicle innovation can give transportation organizations enhanced real-time traffic, travel, and leaving information, to make it simpler to oversee transportation frameworks for diminished traffic as well as blockage.

User applications are very differed, extending from real-time or non-real time streaming of mixed media and intuitive interactions, for example, video-conferencing, climate data or Internet access, for example, information exchange, Web perusing, music download and intelligent recreations, to roadside administration applications, such as, area and value arrangements of eateries or service stations. As a rule, user applications give two essential

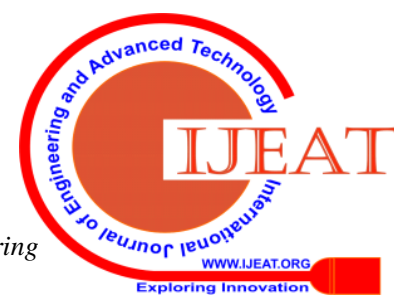


client related administrations: local co-operative services and global internet services. Co-Operative local services are applications concentrating on infotainment which can be acquired from privately based administrations, for example, focal point warning, nearby electronic trade and media downloading. Worldwide internet services. Co-Operative local services are implementations concentrating on infotainment center around information which can be acquired through worldwide Internet administrations. [12]

\section{FUTURE WORK}

The research and development of technologies of IoV will lead to the interrelation of automotive and IT. A more intelligent urban transportation system will be developed, leading to the advancement of social and economic development has a significant effect on the vehicle market, way of life and also methods of conduct. According to study future, IoV work in the Asian-Pacific region will witness rapid growth. It will be a cause for improvement in traffic safety, increasing traffic efficiency and implementing proper control and inspection. Traffic congestion will be lesser and in city parking facilities will be improved.

However, the main objective is to associate multiple users, multiple vehicles and various networks to ensure a connected communication which is reasonable, controllable and trustworthy. Solutions have to be found to make interaction more accessible in a compact city. IoV will be a system which will facilitate excellent systems of transportation without traffic lights, road mishaps and similar issues.

It has become a basic stage with information association among vehicles, people, and road-side infrastructures. It has pulled in a ton of consideration. The idea of IoV is never again a concern of IT applications in the automotive industry; it is now turned into a nationwide and worldwide concern. With the passage of time IoV will end up being a significant part and cause canny transportation systems to manage without traffic lights, street mishaps, and other issues. It also will create a great many individuals to appreciate increasingly helpful, agreeable, and safe traffic administration.

\section{CONCLUSION}

In this paper we have proposed a hybrid IoV architecture, which will improve the data rate, interconnectivity of vehicles, user's location privacy, ensures secure data transfer and better outcome prediction using simple machine learning algorithm. We have referred the idea of Haystack privacy as a security measure, which strengths in privacy strength as more data owners take an interest yet maintain accuracy. The paper has sections about the benefits of IOV, including carbon footprint reduction as one of them. While discussing the applications of IOV, we have mentioned about cooperative adaptive cruise control approach to minimize the number of crash scenario. We have also discussed the probable challenges and future opportunities for researchers and industrialists. We believe seeing an enormous amount of autonomous vehicles soon, which possesses scalable features as proposed in this paper.

\section{REFERENCES}

1. White Paper of Internet of Vehicles (IOV) - Edition 2. Link: https://www.apec.org/-/media/Files/Groups/PPSTI/The-2nd-APEC-W hite-Paper-on-the-Internet-of-Vehicles-Edition-2.pdf

2. Internet of Vehicles and Autonomous Connected Car - Privacy and Security Issues. By Joshua Joy and Mario Gerla, IEEE 2017

3. I. S. Jacobs and C. P. Bean, "Fine particles, thin films and exchange anisotropy," in Magnetism, vol. III, G. T. Rado and H. Suhl, Eds. New York: Academic, 1963, pp. 271-350

4. K. Elissa, "Title of paper if known," unpublished.

5. Nicole, R. "Title of paper with only first word capitalized." J. Name Stand. Abbrev 2 (1987): 740-741.

6. Y. Yorozu, M. Hirano, K. Oka, and Y. Tagawa, "Electron spectroscopy studies on magneto-optical media and plastic substrate interface," IEEE Transl. J. Magn. Japan, vol. 2, pp. 740-741, August 1987 [Digests 9th Annual Conf. Magnetics Japan, p. 301, 1982].

7. M. Young, The Technical Writer's Handbook. Mill Valley, CA: University Science, 1989.

8. Sherazi, H. H. R., Khan, Z. A., Iqbal, R., Rizwan, S., Imran, M. A., \& Awan, K. (2019). A Heterogeneous IoV Architecture for Data Forwarding in Vehicle to Infrastructure Communication. Mobile Information Systems, 2019.

9. I. Al-Anbagi and H. T. Mouftah, "WAVE 4 V2G: wireless access in vehicular environments for vehicle-to-grid applications," Vehicular Communications, vol. 3, pp. 31-42, 2016.

10. J. Huang, "Research on Internet of vehicles and its application in intelligent transportation," Applied Mechanics and Materials, vols. 321-324, 2013, pp. 2818-2821

11. Internet of Vehicles: An Introduction Matthew N. O. Sadiku, Mahamadou Tembely, and Sarhan M. Musa

12. Yang, Fangchun, et al. "An overview of internet of vehicles." China Communications 11.10 (2014): 1-15.

13. Joy, Joshua, and Mario Gerla. "Internet of vehicles and autonomous connected car-privacy and security issues." 2017 26th International Conference on Computer Communication and Networks (ICCCN). IEEE, 2017.

14. Chennikara-Varghese, Jasmine, et al. "Survey of routing protocols for inter-vehicle communications." 2006 Third Annual International Conference on Mobile and Ubiquitous Systems: Networking \& Services. IEEE, 2006

15. Y. Xia and B. Cheng, "A Vehicle Routing Problem based on intelligen batteries transfer management for the EV network," Communications, China, 5, vol. 11, pp. 160-170, 2014

16. V. Namboodiri and G. Lixin, "Prediction-Based Routing for Vehicular $<$ emphasis emphasistype="italic" $>$ Ad Hoc $<$ emphasis $>$ Networks," Vehicular Technology, IEEE Transactions on, 4, vol. 56, pp 2332-2345, 2007.

17. J. Toutouh, J. Garcia-Nieto, and E. Alba, "Intelligent OLSR Routing Protocol Optimization for VANETs," Vehicular Technology, IEEE Transactions on, 4, vol. 61, pp. 1884-1894, 2012.

18. M. Bakhouya, J. Gaber, and P. Lorenz, "An adaptive approach for information dissemination in Vehicular Ad hoc Networks," Journal of Network and Computer Applications, 6, vol. 34, pp. 19711978, 11// 2011

19. J. Nzouonta, N. Rajgure, W. Guiling, and C. Borcea, "VANET Routing on City Roads Using Real-Time Vehicular Traffic Information," Vehicular Technology, IEEE Transactions on, 7, vol. 58, pp. 3609-3626, 2009.

20. H. Xiaoxia and F. Yuguang, "Performance Study of Node-Disjoin Multipath Routing in Vehicular Ad Hoc Networks," Vehicular Technology, IEEE Transactions on, 4, vol. 58, pp. 1942-1950, 2009.

21. M. Fathy, S. GholamalitabarFirouzjaee, and K. Raahemifar, "Improving QoS in VANET Using MPLS," Procedia Computer Science, 0, vol. 10, pp. 1018-1025, // 2012.

22. J. Huang, F. Qian, A. Gerber, Z. M. Mao, S. Sen, and O. Spatscheck, "A close examination of performance and power characteristics of $4 \mathrm{G}$ LTE networks," in Proceedings of 10th International Conference on Mobile Systems, Applications, and Services MobiSys'12, pp. 225-238, Lake District, UK, June 2012. 
23. M. Wellens, B. Westphal, and P. Mahonen, "Performance evaluation of IEEE 802.11-based WLANs in vehicular scenarios," in Proceedings of 2007 IEEE 65th Vehicular Technology Conference-VTC2007-Spring, pp. 1167-1171, IEEE, Dublin, Ireland, April 2007.

24. A. Ghosh, D. R. Wolter, J. G. Andrews, and R. Chen, "Broadband wireless access with WiMax/802.16: current performance benchmarks and future potential," IEEE Communications Magazine, vol. 43, no. 2 , pp. 129-136, 2005.

25. Link:https://scikitlearn.org/stable/modules/generated/sklearn.svm.libs vm.fit.html

26. Dua, D. and Graff, C. (2019). UCI Machine Learning Repository [http://archive.ics.uci.edu/ml]. Irvine, CA: University of California, School of Information and Computer Science.

27. Github Repository for the aforementioned code used, Link: https://github.com/ishanyash/Sensor_SVM

28. C. Dwork and A. Roth, "The algorithmic foundations of differential privacy," Foundations and Trends in Theoretical Computer Science, vol. 9, no. 3-4, pp. 211-407, 2014. [Online]. Available: http: //dx.doi.org/10.1561/0400000042

29. C. Dwork, F. McSherry, K. Nissim, and A. Smith, "Calibrating noise to sensitivity in private data analysis," in TCC, 2006.

30. Andreas Geiger; Martin Lauer; Frank Moosmann; Benjamin Ranft; Holger Rapp; Christoph Stiller; Julius Ziegler (2012). "Team AnnieWAY's Entry to the 2011 Grand Cooperative Driving Challenge". IEEE Transactions on Intelligent Transportation Systems. 13 (3): 1008-1017.

31. Sheikholeslam, S.; Desoer, C. A. (1990). "Longitudinal Control of a Platoon of Vehicles". Proc. of 1990 American Control Conference: 291-296.

32. W. Ejaz and M. Ibnkahla, "Multiband spectrum sensing and resource allocation for IoT in cognitive $5 \mathrm{G}$ networks," IEEE Internet of Things Journal, vol. 5, no. 1, pp. 150-163, 2018.

33. C. Xu, W. Quan, H. Zhang, and L. A. Grieco, "GrIMS: green information-centric multimedia streaming framework in vehicularadhocnetworks,'IEEETransactionsonCircuitsand SystemsforVideoTechnology,vol.28,no.2,pp.483-498,2018.

34. Kate, Rohit J. "Using dynamic time warping distances as features for improved time series classification." Data Mining and Knowledge Discovery 30.2 (2016): 283-312.

35. http://www.cs.ucr.edu/ eamonn/time_series_data/

36. Ben-Hur, Asa; Horn, David; Siegelmann, Hava; and Vapnik, Vladimir N.; "Support vector clustering"; (2001); Journal of Machine Learning Research, 2: 125-137.

\section{AUTHORS PROFILE}

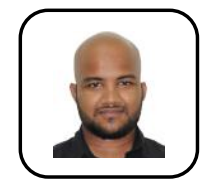

Ishan Yash, Final year undergraduate student from VIT Vellore, Pursuing Electronics engineering with a specialization in Internet of Things and sensors. An aspiring engineer who is interested in data science and analytics.

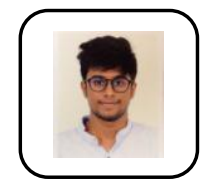

Samir Ahmed Talkal, Final Year undergraduate student from VIT Vellore, Pursuing Information technology engineering. A technocrat having an inclination towards Computer Vision and Machine learning.

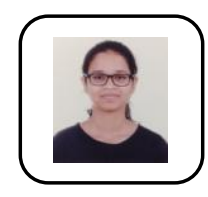

Karapurkar Shivani Prashant, Pre-Final year undergraduate student from VIT, Vellore pursuing Electrical and Electronics Engineering. An aerospace enthusiast having SAE International membership. A hardworking student and an aspiring engineer who wants to achieve goals in the field of electronics 